\title{
Endovascular repair for blunt thoracic aortic injury: 11-year outcomes and postoperative surveillance experience
}

\author{
Konstantinos Spiliotopoulos, MD, ${ }^{\mathrm{a}}$ John Kokotsakis, MD, ${ }^{\mathrm{b}}$ Michalis Argiriou, MD, ${ }^{\mathrm{b}}$ Panagiotis Dedeilias, MD, ${ }^{\mathrm{b}}$ \\ Dimosthenis Farsaris, MD, ${ }^{\mathrm{c}}$ Theodore Diamantis, MD, ${ }^{\mathrm{c}, \mathrm{d}}$ and Christos Charitos, $\mathrm{MD}^{\mathrm{c}, \mathrm{b}}$
}

\begin{abstract}
Objective: Surveillance for patients undergoing thoracic endovascular aortic repair (TEVAR) for blunt thoracic aortic injury (BTAI) varies. Annual chest computed tomographic angiography (CTA) is often recommended but concerns about the risks and costs have emerged. The aim of this study was to examine the optimal follow-up frequency based on 11-year outcomes and surveillance experience.
\end{abstract}

\begin{abstract}
Methods: Seventy-six patients with BTAI received TEVAR from May 2002 to July 2013. Demographics, cardiovascular risk factors, Injury Severity Score (ISS), types, sizes, timing, and outcomes of stent grafts were collected retrospectively.

Results: Mean age was 39.7 years (range, $17-85$ years); 8 (11\%) were women. Mean ISS was $46.2 \pm 18.5$ (deceased, $61.0 \pm 19.2$; surviving, 44.2 $\pm 17.6 ; P=.023$ ). Technical success was achieved in 71 patients $(93.4 \%)$. All-cause mortality was $7(9.2 \%), 1(1.3 \%)$ of which was related to the procedure. Six were lost to follow-up ( $8 \%$ ). To examine the effect of surveillance frequency on outcomes, after excluding the 2 most recent ( $<1$ year) surviving patients, we arbitrarily divided the remaining 61 with stable repairs based on the timing of their follow-up: 36 underwent timely follow-up (within \pm 6 months of the scheduled annual visit; clinical examination, CTA, magnetic resonance angiography, and echocardiography); 25 had delayed follow-up ( $>6$ months after scheduled annual visit). No significant differences were found for survival, graft-related complications, need for reintervention, except for postoperative hypertension, which was higher in the first group. All surviving patients had excellent outcomes, with no cerebrovascular accidents, paraplegia, or paraparesis; the median follow-up for both groups was 3 years (interquartile range 2.0-3.5, 1.5-5.4 years).
\end{abstract}

Conclusions: Midterm outcomes of TEVAR for patients with stable repair after BTAI are excellent, both with timely (1.0-1.5 years) and delayed ( $>1.5$ years) follow-up intervals after a median surveillance period of 3 years. A larger prospective randomized study could lead to a more relaxed, but equally safe surveillance schedule for these patients, lowering risks and costs. (J Thorac Cardiovasc Surg 2014;148:2956-61)

See related commentry on page 2962.

In the previous report ${ }^{1}$ from our institution, we published our short-term results comparing the early use of thoracic endovascular aortic repair (TEVAR) for patients with blunt thoracic aortic injury (BTAI) in the proximal descending aorta with open repair. Now that TEVAR is arguably the standard of care for these patients, the number of cases has

\footnotetext{
From the Department of Cardiothoracic Surgery, ${ }^{\text {a }}$ Stanford University Medical Center, Stanford, Calif; Cardiothoracic Surgery, ${ }^{b}$ and Interventional Radiology, Evaggelismos General Hospital, and Department of Surgery, ${ }^{\mathrm{d}}$ National and Kapodistrian University of Athens, Athens, Greece.

Disclosures: Authors have nothing to disclose with regard to commercial support.

Read at The American Association for Thoracic Surgery Aortic Symposium, New York, New York, April 24-25, 2014.

Received for publication April 21, 2014; revisions received July 30, 2014; accepted for publication Aug 7, 2014; available ahead of print Sept 17, 2014.

Address for reprints: Konstantinos Spiliotopoulos, MD, Department of Cardiothoracic Surgery, Stanford University Medical Center, Falk Cardiovascular Research Center, 300 Pasteur Drive, Stanford, CA 94305-5407 (E-mail: kspiliot@stanford.edu).

$0022-5223 / \$ 36.00$

Copyright (c) 2014 by The American Association for Thoracic Surgery

http://dx.doi.org/10.1016/j.jtcvs.2014.08.019
}

increased and the midterm results reported in multiple centers are encouraging. ${ }^{2,3}$

The optimal method of repair is still unknown and the postoperative surveillance for patients undergoing TEVAR for BTAI is still under discussion. Open repair is more invasive, but interval posteroanterior and lateral chest radiographs may constitute adequate postoperative surveillance. Conversely, although TEVAR is less invasive, surveillance imaging, usually annually, requires significantly greater expenditure on resources and exposes patients to the toxicity of intravenous contrast agents and the risks of radiation. BTAI is more common in relatively young people, therefore these concerns are even stronger in this subset of patients. This report seeks to demonstrate the safety of less frequent imaging in patients with stable repairs, based on our outcomes and postoperative surveillance experience with 76 patients over an 11-year period.

\section{PATIENTS AND METHODS}

We retrospectively reviewed the records of 76 consecutive patients who were referred to our tertiary trauma center from May 2002 to July 2013 with BTAI, either isolated or with associated injuries in other systems, and who received a thoracic aortic stent graft during treatment. To our 


\section{Abbreviations and Acronyms \\ BTAI = blunt thoracic aortic injury \\ $\mathrm{CT}=$ computed tomography \\ CTA $=$ computed tomographic angiography \\ $\mathrm{CVA}=$ cerebrovascular accident \\ $\mathrm{IQR}=$ interquartile range \\ ISS $=$ Injury Severity Score \\ LSA $=$ left subclavian artery \\ $\mathrm{RCT}=$ randomized control trial \\ RFA $=$ right femoral artery \\ TEVAR $=$ thoracic endovascular aortic repair}

knowledge, this is the largest series of TEVARs for patients with BTAI published from a single institution. The Scientific and Ethics Committee of our hospital approved the study. Seventy-two patients $(94.7 \%)$ had been in a motor vehicle accident; 4 patients $(5.3 \%)$ had fallen accidentally or suicidally and their aortic injury was the result of sudden deceleration. All patients had aortic injury confirmed initially by a chest computed tomography (CT) scan at 7-mm slice thickness, followed by computed tomographic angiography (CTA) at $1 / 0.8 \mathrm{~mm}$ and reconstruction in 3 planes (axial, coronal, and sagittal) in cases of a proximal landing zone less than $0.8 \mathrm{~cm}$, in order to accurately define the feasibility of TEVAR. Medical management was applied until definitive endovascular repair. The types of thoracic stent grafts used were the Talent $(n=13)$ and Valiant $(n=42)$ (Medtronic, Inc, Minneapolis, Minn), the Relay $(n=20)$ (Bolton Medical, Sunrise, Fla), and the E-Vita Thoracic $(\mathrm{n}=1)$ (Jotec, Hechingen, Germany). All patients were managed in the angiographic suite under local anesthesia, unless the patients were sedated because of concomitant injuries (mainly head injuries). Stent grafts were delivered through the femoral artery, after a cut-down exposure, with a $22 \mathrm{~F}$ to $25 \mathrm{~F}$ delivery system, and digital aortography was performed through the left brachial artery for injury confirmation and stent guidance. The delivery system was promoted over an extra stiff wire guide (Lunderquist; Cook Medical, Inc, Bloomington, Ind), with its flexible tip previously placed just distally to the aortic valve through a $5 \mathrm{~F}$ angiographic catheter (IMPRESS Vertebral, Merit Medical Systems, Inc, South Jordan, Utah). We used stent graft oversizing of $10 \%-15 \%$ compared with the aortic diameter on the preoperative CTA. We believe that oversizing is appropriate for the healthy aortic wall of patients with BTAI, only when adjacent dissection is not present. If dissection coexists in these patients (a relatively rare entity), we avoid oversizing. Heparin (5000 IU intravenous bolus) was given only in lengthy procedures, but was avoided in cases of excessive bleeding from other anatomic sites or if fast deployment was achieved. Also, $40 \mathrm{~mL}$ of normal saline was administered to the delivery system before the stent graft insertion to minimize the porosity phenomenon (possibility of a transgraft endoleak, type 4, immediately after deployment). Local papaverine was applied on the arterial site of insertion (ie, the femoral artery) in cases of hemodynamic instability to enable the access. In some young patients, in whom femoroiliac access seemed limited in caliber, we have always found that their arterial wall elasticity allowed for safe insertion of the delivery system in a glove over a hand manner. However, if insertion had not been deemed feasible or safe, an appropriate iliac or aortic conduit graft could have been used. Digital subtraction angiography was performed on completion to evaluate correct placement of the device, patency, and absence of endoleaks. The role of the surgeons in the procedure was to provide open exposure (cut-down) of the femoral artery and facilitate the insertion of the delivery system; all of the actual 76 implantations were done by the interventional radiologists.
We retrospectively analyzed the data on the patients' demographics, preoperative cardiovascular risk factors (hypertension, diabetes mellitus, hyperlipidemia, and smoking), comorbidities and previous cardiothoracic surgery procedures, mode and anatomic extent of aortic injury, concomitant injuries and Injury Severity Score (ISS), time between the accident and the procedure, the type and size of stent grafts used, the potential left subclavian artery (LSA) orifice coverage, and any subsequent need for revascularization. Perioperative and postoperative outcomes were also reviewed as follows:

(1) Technical success rate and the need for conversion to an open procedure, immediate type 1 endoleak and procedure-related vascular injury, length of hospital stay, stroke, paraplegia, and all-cause and stent graft-related mortality within the index hospital admission.

(2) Mid- and long-term graft-related sequelae (chest tightness, persistent hypertension and fever), the position, integrity and potential presence of endoleaks on the follow-up imaging (ie, CTA) and the need for reintervention.

\section{Statistical Analysis}

Quantitative variables are expressed as mean values (standard deviation) or as median values (interquartile range [IQR]). Qualitative variables are expressed as absolute and relative frequencies. The $\chi^{2}$ and Fisher exact tests were used for comparison of proportions. The Student $t$ test or Mann-Whitney test was computed for comparison of the mean values. Life table analyses were used to calculate cumulative survival (standard errors) for specific time intervals. Kaplan-Meier survival estimates were graphed over the follow-up period (Figure 1). The multiple Cox proportional-hazard model was used in a stepwise method ( $P$ for removal was set at .1 and $P$ for entry was set at .05) to determine independent factors associated with survival. The assumption of proportional hazards was evaluated by testing for interaction with a continuous time variable. All reported $P$ values are 2 -tailed. The statistical analyses were conducted using SPSS version 18.0 (SPSS, Inc, Chicago, Ill)

\section{RESULTS}

The mean age of the patient cohort was 39.7 years (range, 17-85 years) and 8 of the 76 patients (11\%) were women (Table 1). One patient had recently ( 5 months before) undergone an open aortic valve replacement. Mean ISS was $46.2 \pm 18.5$; the ISS was significantly different between deceased $(61.0 \pm 19.2)$ and surviving patients $(44.2 \pm 17.6 ; P=.023)$. In all patients treated with TEVAR, BTAI was associated with intimal flaps and/or thrombus, intramural or periadventitial hematomas, and/or pseudoaneurysms in the vicinity of the isthmus (BTAI classification, grades I-III). ${ }^{4}$ Eighteen patients $(23.7 \%)$ underwent a delayed TEVAR, more than 24 hours after injury, either because of other concomitant life-threatening injuries requiring immediate intervention (eg, emergency laparotomy or craniotomy) or delayed arrival at our center. These patients were managed medically with antihypertensive and counterpulsation medications until they received definitive treatment with TEVAR. Primary technical success was achieved in 71 patients $(93.4 \%)$. Five patients $(6.6 \%)$ had a type 1 endoleak, recognized immediately, and 4 of them required another stent graft (cuff); 1 patient, who was a Jehovah witness, had a severe aortic injury associated 


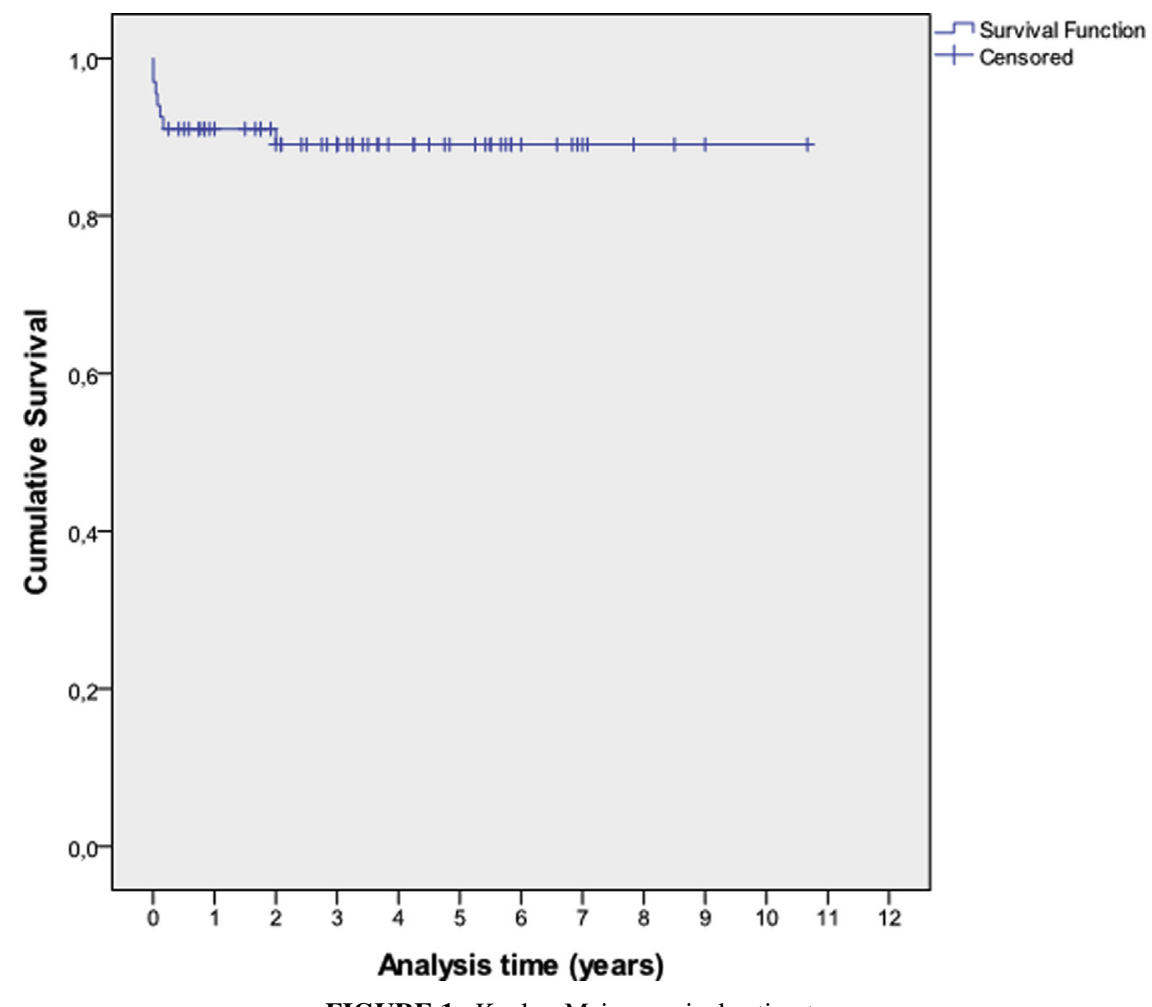

FIGURE 1. Kaplan-Meier survival estimates.

with pseudoaneurysm and overt rupture, and developed profound hemodynamic instability 14 hours postoperatively and died in the angiographic suite due to exsanguination without any possibility for additional stent grafting. None of the patients had a conversion to open procedure. Our strategy for the proximal landing zone was as follows: $2 \mathrm{~cm}$ if the distance from the LSA orifice was greater than $2.0 \mathrm{~cm}$, partial coverage of the LSA orifice if the distance from the LSA orifice was 1.5 to $2.0 \mathrm{~cm}$, and full coverage of the LSA orifice if the distance was less than $1.5 \mathrm{~cm}$. Eleven patients had their LSA orifice covered by the stent graft, but only 2 patients with clinical ischemia of the left arm were revascularized with carotid-subclavian bypass grafting. One of them had an aberrant left vertebral artery originating from the aortic arch. The median length of stay was 15.5 days (IQR, 8.5-32 days). Two patients had complications from the stent graft insertion site, the right femoral artery (RFA); in the first case, the artery was occluded, but the patient was treated conservatively and had a successful recovery. The second patient was readmitted with a pseudoaneurysm in the RFA, which was successfully drained and primarily repaired. All-cause mortality within the index hospital admission was $9.2 \%$ (7 patients) and procedure-related mortality was $1.3 \%$ (the patient with a type 1 endoleak who died in the angiographic suite). None of the treated patients experienced any procedure-related cerebrovascular accident (CVA) or paraplegia/paraparesis. Kaplan-Meier survival estimates are presented in Figure 1. The cumulative event-free rate at 1 month was $90.0 \%$ (standard error $=$ $4.0 \%$ ); at 6 months, 1,5 , and 10 years, it was equal to $88.0 \%$ (standard error $=4.0 \%$ ). Multipe Cox regression analysis in a stepwise method showed that increased age (hazard ratio $=1.04 ; 95 \%$ confidence interval, 1.01-1.08) and increased ISS (hazard ratio $=1.06 ; 95 \%$ confidence interval, 1.01-1.11) were independently associated with greater hazard. Only 6 patients $(8 \%)$ were lost to followup, which differentiates this report from almost all others. This study's follow-up closing window was between March 2013 and September 2013.

During the same 11-year period (May 2002 to July 2013), only 8 patients underwent open surgical repair with synthetic graft interposition for BTAI in our institution; the last one was performed in August 2004 (BTAI from a fall).

To examine the effect of follow-up timing on mid- and long-term outcomes, we focused on adherence to postoperative follow-up by eligible patients. First, we excluded the 7 deceased patients, the 6 patients lost to follow-up and the 2 most recent $(<1$ year) surviving patients. We then arbitrarily divided the remaining 61 patients, all of whom had stable repairs, with an adequate proximal landing zone and no bird-beak appearance, into 2 groups, based on the timing of their follow-up. The first group consisted of 36 patients with a generally timely follow-up (clinical examination and CTA/MRA/echocardiography) within 
TABLE 1. Demographics and clinical characteristics of the entire cohort of patients

\begin{tabular}{|c|c|}
\hline Characteristic & n $(\%)$ \\
\hline \multicolumn{2}{|l|}{ Sex } \\
\hline Male & $68(89.5)$ \\
\hline Female & $8(10.5)$ \\
\hline Mean age, y (SD) & $39.7(17.1)$ \\
\hline \multicolumn{2}{|l|}{ Mode of injury } \\
\hline MVA & $72(94.7)$ \\
\hline Fall & $4(5.3)$ \\
\hline ISS, mean (SD) & $46.2(18.5)$ \\
\hline \multicolumn{2}{|l|}{ Delayed ( $>24 \mathrm{~h}$ ) TEVAR } \\
\hline No & $58(76.3)$ \\
\hline Yes & $18(23.7)$ \\
\hline \multicolumn{2}{|l|}{ LSA orifice covered } \\
\hline No & $65(85.5)$ \\
\hline Yes & $11(14.5)$ \\
\hline \multicolumn{2}{|l|}{ Primary technical success } \\
\hline No & $5(6.6)$ \\
\hline Yes & $71(93.4)$ \\
\hline \multicolumn{2}{|l|}{ Conversion to open procedure } \\
\hline No & $76(100)$ \\
\hline Yes & $0(0)$ \\
\hline \multicolumn{2}{|l|}{ Endoleak type I (immediate) } \\
\hline No & $71(93.4)$ \\
\hline Yes & $5(6.6)$ \\
\hline \multicolumn{2}{|l|}{ CVA } \\
\hline No & $76(100)$ \\
\hline Yes & $0(0)$ \\
\hline \multicolumn{2}{|l|}{ Paraplegia } \\
\hline No & $76(100)$ \\
\hline Yes & $0(0)$ \\
\hline \multicolumn{2}{|l|}{ Vascular access complications } \\
\hline No & $74(97.4)$ \\
\hline Yes & $2(2.6)$ \\
\hline Median length of stay, $d$ (IQR) & $15.5(8.5-32)$ \\
\hline All-cause index mortality & 7 (9.2) \\
\hline Graft-related mortality & $1(1.3)$ \\
\hline Median follow-up, mo (IQR) & $38(25-66)$ \\
\hline
\end{tabular}

$S D$, Standard deviation; $M V A$, motor vehicle accident; ISS, Injury Severity Score; TEVAR, thoracic endovascular aortic repair; LSA, left subclavian artery; CVA, cerebrovascular accident; $I Q R$, interquartile range.

6 months of the scheduled yearly visit and the second group consisted of 25 patients with nontimely, delayed follow-up ( $>6$ months after their scheduled yearly visit). There were no significant differences between the 2 groups, except from persistent hypertension, which was more prevalent in the timely follow-up group (Table 2). All patients had excellent outcomes, with no major stent graft-related adverse events, no CVAs or paraplegia/ paraparesis, or need for reintervention at a median follow-up of 3 years for both groups (IQR, 2.0-3.5 years and 1.5-5.4 years, respectively). One patient from the timely follow-up group was found to have a bicuspid aortic valve and a 5.5-cm ascending aorta aneurysm 15 months postoperatively and he had his ascending aorta replaced.
One patient in the delayed follow-up group died at 5 years due to unrelated causes.

\section{DISCUSSION}

Twenty-two years after the first TEVAR implantation by Dake and Miller ${ }^{5}$ at Stanford, in a patient with a postoperative enlarging descending thoracic aortic false aneurysm, dissemination of this biotechnological advent has been extensive, as has progress in the construction and deployment method of stent grafts, with a resulting decrease in periprocedural morbidity and mortality. The advent of the new hybrid operating rooms, with ample use of adequate, contemporary radiology equipment, working space and support, will further streamline major endovascular procedures, making them both safer and easier to perform.

Aneurysmal disease is the most frequent indication for endovascular aortic repair in the abdominal aorta, but there are multiple pathologies of the thoracic aorta that can be treated with TEVAR, such as aneurysms, dissections, and traumatic injuries (BTAI).

The surveillance focus and frequency of follow-up for patients after TEVAR should be dependent on the type of underlying pathology. In the setting of BTAIs, patients are usually young or middle-aged without any preexisting aortic diseases. Theoretically, once excluded, the traumatic aortic pseudoaneurysm in these patients should thrombose and the otherwise normal aorta should heal without any physiologic predisposition to further degeneration. On the other hand, young patients usually have a more tightly angulated aortic arch, which if combined with excessive oversizing on a healthy, stiff aortic wall, could predispose to device infolding and collapse. Thus, the most important focus of the follow-up protocol in this case should be the position and integrity of the stent graft device, rather than size changes, branch patency, entry/reentry of false channels and endoleaks, as in the case of aneurysms and dissections. However, the frequency of postoperative surveillance in this subgroup of patients (TEVAR after BTAI) is still under discussion and remains empirically annual in many centers, given the lack of evidence from prospective randomized control trials (RCTs).

Moreover, postoperative surveillance in this subcohort of patients after TEVAR is a challenging task. Young, healthy adults, with sometimes severe concomitant injuries, tend to neglect the follow-up protocol of an endovascular procedure, which was executed with minimal perioperative disturbance, despite concerns about the long-term efficacy of this novel treatment. We actually had to phone patients in the delayed follow-up group several times to persuade them to visit the hospital for their clinical and imaging reexamination. An interesting finding of our retrospective analysis was that, among the group of patients with a timely surveillance schedule, a significantly higher percentage had 
TABLE 2. Demographics, clinical characteristics, and outcomes of systematic and nonsystematic follow-up patients groups

\begin{tabular}{|c|c|c|c|}
\hline & \multicolumn{2}{|c|}{$\begin{array}{c}\text { Systematic follow-up, } \\
\text { n ( } \%)\end{array}$} & \multirow[b]{2}{*}{$P$} \\
\hline & No $(N=25)$ & Yes $(\mathbf{N}=\mathbf{3 6})$ & \\
\hline \multicolumn{4}{|l|}{ Sex } \\
\hline Male & $22(88.0)$ & $32(88.9)$ & $1.000 \|$ \\
\hline Female & $3(12.0)$ & $4(11.1)$ & \\
\hline Mean age, y (SD) & $40.4(15.0)$ & $37.4(17.3)$ & $.483 \ddagger$ \\
\hline \multicolumn{4}{|l|}{ Mode of injury } \\
\hline MVA & $25(100.0)$ & $33(91.7)$ & $.262 \|$ \\
\hline Fall & $0(0.0)$ & $3(8.3)$ & \\
\hline \multicolumn{4}{|l|}{ Cardiovascular risk factors } \\
\hline \multicolumn{4}{|l|}{ Smoking } \\
\hline No & $14(56.0)$ & $23(63.9)$ & $.535^{*}$ \\
\hline Yes & $11(44.0)$ & $13(36.1)$ & \\
\hline \multicolumn{4}{|l|}{ Hypertension } \\
\hline No & $21(84.0)$ & $26(72.2)$ & $.282 \|$ \\
\hline Yes & $4(16.0)$ & $10(27.8)$ & \\
\hline \multicolumn{4}{|l|}{ Hyperlipidemia } \\
\hline No & $23(92.0)$ & $29(80.6)$ & $.286 \|$ \\
\hline Yes & $2(8.0)$ & $7(19.4)$ & \\
\hline \multicolumn{4}{|l|}{ Diabetes mellitus } \\
\hline No & $24(96.0)$ & $35(97.2)$ & $1.000 \|$ \\
\hline Yes & $1(4.0)$ & $1(2.8)$ & \\
\hline ISS, mean (SD) & $40.1(17.2)$ & $33.4(15.9)$ & $.133 \ddagger$ \\
\hline \multicolumn{4}{|l|}{ Clinical characteristics } \\
\hline \multicolumn{4}{|l|}{ Stent grafts used } \\
\hline Talent (Medtronic) & 4 & 5 & $>.05$ \\
\hline Valiant (Medtronic) & 14 & 25 & $>.05$ \\
\hline Relay (Bolton Medical) & 6 & 6 & $>.05$ \\
\hline E-Vita Thoracic (Jotec) & 1 & 0 & $>.05$ \\
\hline $\begin{array}{l}\text { Stent graft size (mean), } \\
\text { mm (SD) }\end{array}$ & $\begin{array}{l}33.9(4.1) \times \\
133.2(21.4)\end{array}$ & $\begin{array}{l}32.6(3.4) \times \\
128.2(24.2)\end{array}$ & $>.05$ \\
\hline \multicolumn{4}{|l|}{ Delayed ( $>24 \mathrm{~h}$ ) TEVAR } \\
\hline No & $19(76.0)$ & $25(69.4)$ & $.574 *$ \\
\hline Yes & $6(24.0)$ & $11(30.6)$ & \\
\hline \multicolumn{4}{|l|}{ LSA orifice covered } \\
\hline No & $24(96.0)$ & $29(80.6)$ & $.125 \|$ \\
\hline Yes & $1(4.0)$ & $7(19.4)$ & \\
\hline \multicolumn{4}{|l|}{ Primary technical success } \\
\hline No & $3(12.0)$ & $1(2.8)$ & $.296 \|$ \\
\hline Yes & $22(88.0)$ & $35(97.2)$ & \\
\hline \multicolumn{4}{|c|}{ Conversion to open procedure } \\
\hline No & $25(100.0)$ & $36(100.0)$ & $-\dagger$ \\
\hline Yes & $0(0.0)$ & $0(0.0)$ & \\
\hline \multicolumn{4}{|l|}{ Endoleak type I (immediate) } \\
\hline No & $22(88.0)$ & $35(97.2)$ & $.296 \|$ \\
\hline Yes & $3(12.0)$ & $1(2.8)$ & \\
\hline \multicolumn{4}{|c|}{ Postoperative cerebrovascular accident } \\
\hline No & $25(100.0)$ & $36(100.0)$ & 1.000 \\
\hline Yes & $0(0.0)$ & $0(0.0)$ & \\
\hline \multicolumn{4}{|c|}{ Postoperative paraplegia/paraparesis } \\
\hline No & $25(100.0)$ & $36(100.0)$ & 1.000 \\
\hline Yes & $0(0.0)$ & $0(0.0)$ & \\
\hline \multicolumn{4}{|c|}{ Vascular access complications } \\
\hline No & $24(96.0)$ & $35(97.2)$ & $1.000 \|$ \\
\hline
\end{tabular}

TABLE 2. Continued

\begin{tabular}{|c|c|c|c|}
\hline & \multicolumn{2}{|c|}{$\begin{array}{c}\text { Systematic follow-up, } \\
\text { n (\%) } \\
\end{array}$} & \multirow[b]{2}{*}{$\boldsymbol{P}$} \\
\hline & No $(\mathbf{N}=\mathbf{2 5})$ & Yes $(N=36)$ & \\
\hline Yes & $1(4.0)$ & $1(2.8)$ & \\
\hline $\begin{array}{l}\text { Median length of stay, } \\
\text { d (IQR) }\end{array}$ & $11.5(5-26)$ & $17.5(10-38.5)$ & $.106 \S$ \\
\hline \multicolumn{4}{|l|}{ Graft-related complications } \\
\hline \multicolumn{4}{|l|}{ Fever } \\
\hline No & $25(100.0)$ & $33(91.7)$ & $.262 \|$ \\
\hline Yes & $0(0.0)$ & $3(8.3)$ & \\
\hline \multicolumn{4}{|l|}{ Hypertension } \\
\hline No & $23(92.0)$ & $18(50.0)$ & $.001 *$ \\
\hline Yes & $2(8.0)$ & $18(50.0)$ & \\
\hline \multicolumn{4}{|l|}{ Chest tightness } \\
\hline No & $22(88.0)$ & $32(88.9)$ & 1.000 \\
\hline Yes & $3(12.0)$ & $4(11.1)$ & \\
\hline $\begin{array}{l}\text { Median follow-up, } \\
\text { y (IQR) }\end{array}$ & $3(1.5-5.4)$ & $3(2-3.5)$ & 1.000 \\
\hline \multicolumn{4}{|l|}{ Mortality (>1.5 y) } \\
\hline No & $24(96.0)$ & $36(100.0)$ & .410 \\
\hline Yes & $1(4.0)$ & $0(0.0)$ & \\
\hline
\end{tabular}

$S D$, Standard deviation; $M V A$, motor vehicle accident; ISS, Injury Severity Score; $T E V A R$, thoracic endovascular aortic repair; $L S A$, left subclavian artery; $I Q R$, interquartile range. *Pearson $\chi^{2}$ test. $\dagger$ Not computed because of no distribution.

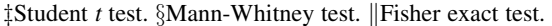

persistent hypertension. An explanation could be that symptomatic hypertension had potentially alerted these patients to the significance of regular surveillance.

Although concerns have recently been raised about effective patient selection and appropriate use of TEVAR for different aortic pathologies and patient subsets, ${ }^{6}$ consensus exists, based on observational evidence rather than prospective RCTs, that BTAI is one of the most effective indications for its use, which also coincides with our 11-year experience and postoperative surveillance of these patients.

However, we realized that in patients with stable repair, outcomes did not differ much between those with regular and those with irregular follow-up schedules, which ultimately prompted us to launch this retrospective study. The extent of aortic injury and the mix of stent graft types and sizes used in those 2 groups were comparable, as was their last follow-up time period and their demographic characteristics, the timing of the intervention, and their preexisting cardiovascular risk factors and ISS. To our knowledge, this is the first attempt to analyze data on postoperative surveillance for patients with BTAI treated with TEVAR.

\section{Limitations}

Two important limitations of our study are the moderate number of patients, which undermines the statistical power of the results, and the fact that it is retrospective, which 
allows for major selection bias. Moreover, we acknowledge the lack of longer-term follow-up data in establishing the safety and efficacy of TEVAR; our study cannot provide them because the median follow-up for both groups was 3 years. Potential graft-related complications include material failure (eg, stent fractures and fabric fatigue; the nitinol in certain stent grafts on bench testing is expected to last only about 10 years), recurrent endoleaks, pseudoaneurysm formation, and stent graft migration, collapse, stenosis, or thrombosis. The only way to identify any such issues early enough would be regular and systematic surveillance. Our results can only offer some insight into the first 1 to 3.5 years of postoperative follow-up (IQR of the timely follow-up group, which is included in the IQR of the nontimely group).

However, it is important to initiate a scientific discussion regarding the optimal frequency of surveillance for these patients for the following reasons.

First, CT scans are not as safe as we might think ${ }^{7}$ and because they are still the preferred imaging modality for the follow-up of patients with BTAI treated with TEVAR, they represent a significant amount of radiation exposure, especially for the young trauma population, which can lead to severe complications, such as radiation burns, ulceration, renal toxicity by intravenous contrast agents and, most important, the development of malignancies. A calculation of the actual radiation doses delivered by commonly performed CT studies (including chest CT) and quantification of the associated cancer risks using the National Research Council's models found that the risk of cancer from a single CT scan could be as high as 1 in $80 .^{8}$ If this risk is multiplied by several times from CT surveillance in the lifetime of a young patient treated with TEVAR after BTAI, the resulting cumulative risk may become prohibitively high.

Second, the cost of this intensive yearly follow-up schedule will soon become a major issue for the budget of hospitals and health systems, because the number of young trauma patients will continue to increase. Concerns about the uncritical use and cost of high-tech medical imaging have already emerged, placing imaging in the policy spotlight and solidifying the general agreement that a substantial number of imaging examinations are unnecessary and do not positively contribute to patient care. ${ }^{9}$

In conclusion, midterm outcomes of TEVAR for patients with stable repair after BTAI are excellent, both with timely (1.0-1.5 years) and delayed ( $>1.5$ years) follow-up intervals at a median surveillance of 3 years. However, we need large, multicenter, randomized studies to establish a more realistic, less intensive, but equally safe surveillance schedule for carefully selected patients with stable repairs, which would help to substantially reduce the associated risks and costs.

The corresponding author would like to gratefully acknowledge the substantial contribution of the following individuals in reviewing, editing and commenting on this article before submission: Michael D. Dake, MD, Thelma and Henry Doelger Professor; D. Craig Miller, MD, Thelma and Henry Doelger Professor; and R. Scott Mitchell, MD, Professor Emeritus, Department of Cardiothoracic Surgery, Stanford University Medical Center, Standford, Calif.

\section{References}

1. Kokotsakis J, Kaskarelis I, Misthos P, Athanasiou T, Kanakakis K, Athanasiou C, et al. Endovascular versus open repair for blunt thoracic aortic injury: short-term results. Ann Thorac Surg. 2007;84:1965-70.

2. Lee WA, Matsumura JS, Mitchell RS, Farber MA, Greenberg RK, Azizzadeh A et al. Endovascular repair of traumatic thoracic aortic injury: clinical practice guidelines of the Society for Vascular Surgery. J Vasc Surg. 2011;53: 187-92.

3. Demetriades D, Velmahos GC, Scalea TM, Jurkovich GJ, Karmy-Jones R, Teixeira PG, et al., American Association for the Surgery of Trauma Thoracic Aortic Injury Study Group. Operative repair or endovascular stent graft in blunt traumatic thoracic aortic injuries: results of an American Association for the Surgery of Trauma Multicenter Study. J Trauma. 2008;64:561-71.

4. Azizzadeh A, Keyhani K, Miller CC 3rd, Coogan SM, Safi HJ, Estrera AL. Blunt traumatic aortic injury: initial experience with endovascular repair. J Vasc Surg. 2009;49:1403-8.

5. Dake MD, Miller DC, Semba CP, Mitchell RS, Walker PJ, Liddell RP. Transluminal placement of endovascular stent/grafts for the treatment of descending thoracic aortic aneurysms. N Engl J Med. 1994;331:1729-34.

6. Miller DC. Through the looking glass: the first 20 years of thoracic aortic stent-grafting. J Thorac Cardiovasc Surg. 2013;145:142-8.

7. Smith-Bindman R. Is computed tomography safe? N Engl J Med. 2010;363:1-4.

8. Smith-Bindman R, Lipson J, Marcus R, Kim KP, Mahesh M, Gould R, et al Radiation dose associated with common computed tomography examinations and the associated lifetime attributable risk of cancer. Arch Intern Med. 2009; 169:2078-86

9. Hillman BJ, Goldsmith JC. The uncritical use of high-tech medical imaging N Engl J Med. 2010;363:4-6. 